\title{
Simulating dislocation loop internal dynamics and collective diffusion using stochastic differential equations
}

\author{
P. M. Derlet* \\ Condensed Matter Theory Group, Paul Scherrer Institut, CH-5232 Villigen PSI, Switzerland
}

M. R. Gilbert and S. L. Dudarev

EURATOM/CCFE Fusion Association, Culham Centre for Fusion Energy, Oxfordshire OX14 3DB, United Kingdom

(Received 29 July 2011; revised manuscript received 29 September 2011; published 20 October 2011)

\begin{abstract}
Nanoscale prismatic loops are modeled via a partial stochastic differential equation that describes an overdamped continuum elastic string, with a view to describing both the internal and collective dynamics of the loop as a function of temperature. Within the framework of the Langevin equation, expressions are derived that relate the empirical parameters of the model, the friction per unit length, and the elastic stiffness per unit length, to observables that can be obtained directly via molecular-dynamics simulations of interstitial or vacancy prismatic loop mobility. The resulting expressions naturally exhibit the properties that the collective diffusion coefficient of the loop (i) scales inversely with the square root of the number of interstitials, a feature that has been observed in both atomistic simulation and in situ TEM investigations of loop mobility, and (ii) the collective diffusion coefficient is not at all dependent on the internal interactions within the loop, thus qualitatively rationalizing past simulation results showing that the characteristic migration energy barrier is comparable to that of a single interstitial, and cluster migration is a result of individual (but correlated) interstitial activity.
\end{abstract}

DOI: 10.1103/PhysRevB.84.134109

PACS number(s): 61.72.-y, 61.80.-x, 61.82.Bg

\section{INTRODUCTION}

Materials in extreme radiation environments exhibit a complex and dynamic behavior strongly influenced by their initial as-prepared microstructure, and at the same time show fundamental generic features-radiation defects are mobile, interact with each other via long-range elastic forces and, when sufficiently close, react and transform to microstructures characterized by quite different mobility and interaction laws. Collectively, such defects evolve and interact with the mobile dislocations and thus strongly affect the mechanical response of the material, where, for example, stable and immobile (possibly extended) defects evolve to cause hardening and a resulting loss in ductility.

$A b$ initio and empirical atomistic simulations have provided considerable insight into the microscopic aspects of microstructural evolution, ${ }^{1}$ through the calculation of (zerotemperature) formation and migration energies of individual self-interstitial atom (SIA) and vacancy defects. ${ }^{2-4}$ When the migration energies are sufficiently small, empirical atomistic simulations performed at finite temperature have allowed for the direct measurement of defect diffusion coefficients demonstrating that individual SIAs have nontrivial mobility properties that strongly depend on material type and temperature. For the nonmagnetic bcc transition metals the ground-state structure of a single SIA is a $\langle 111\rangle$ crowdion, ${ }^{5}$ in which the extra atom is delocalized within a $\langle 111\rangle$ string over a number of lattice constants. On the other hand, for ferromagnetic bcc $\mathrm{Fe}$ it is the $\langle 110\rangle$ dumbbell, which is a somewhat more localized defect. These quite different structures admit different thermally activated mechanisms for SIA transport.

For the case of the $\langle 111\rangle$ crowdion, atomistic simulation work has revealed the occurrence of fast one-dimensional (1D) diffusion along the $\langle 111\rangle$ direction with each component migration involving several interatomic distances. ${ }^{6,7} \mathrm{~A}$ high value of the diffusion coefficient reflects a low value of the so-called lattice friction, where $D=k_{\mathrm{B}} T / \gamma$ in which $D$ is the diffusion constant, $\gamma$ is the friction coefficient, and $T$ is the temperature. In terms of a Frenkel-Kontorova model for a generic interstitial, ${ }^{8,9}$ such SIA activity could be interpreted as a manifestation of the formation of a true quasiparticle "whose properties are radically different from properties of (the) strongly interacting atoms forming that quasiparticle," and whose friction coefficient exhibits a temperature dependence that results in a non-Arrhenius behavior for the corresponding diffusion coefficient. This theoretical prediction was later confirmed using atomistic simulation with the observation of non-Arrhenius behavior of crowdion SIAs in vanadium..$^{10}$ Indeed for the case of tungsten, both simulation and theory have shown that the diffusion coefficient of the $\langle 111\rangle$ crowdion obeys a linear temperature dependence in the high-temperature regime due to a corresponding temperatureindependent friction coefficient. ${ }^{11}$ For the case of bcc Fe, the ground-state $\langle 110\rangle$ interstitial dumbbell executes 3D motion via the Johnson mechanism ${ }^{12}$ resulting in a nearest-neighbor displacement and rotation of the dumbbell to another $\langle 110\rangle$ direction. With increasing temperature, simulation has also found that the $\langle 110\rangle$ dumbbell structure is excited to a $\langle 111\rangle$ configuration with subsequent 1D movement over many interatomic distances until relaxation to a $\langle 110\rangle$ dumbbell configuration of a possibly different orientation. ${ }^{13,14}$

Atomistic simulations have also investigated the stability and mobility of clusters of SIAs in which the SIAs condense to a $\langle 111\rangle$ platelet of extra atoms. ${ }^{6,7,15-18}$ Such a $\langle 111\rangle$ configuration also occurs in bcc $\mathrm{Fe}$ for greater-than-three SIA clusters, ${ }^{14,19-21}$ despite the ground-state structure of a single SIA being the $\langle 110\rangle$ dumbbell interstitial. At even higher temperatures for bcc Fe, the ground-state prismatic loop structure has a $\langle 100\rangle$ Burgers vector and is immobile in the time 
frame of atomistic simulation, an experimental observation that is now understood to arise from magnetic fluctuations, which also leads to the $\alpha-\gamma$ structural phase transformation in $\mathrm{Fe}^{22}$ This aspect will not be considered further in the present work. For the case of vanadium SIA clusters, simulation has also demonstrated strong non-Arrhenius behavior which was analogous to that seen for a single SIA. ${ }^{18}$ All such work demonstrated that in terms of the jump frequency ${ }^{23}$ (rather than the diffusion coefficient), Arrhenius behavior is apparent, giving two important results: (1) the energy barrier associated with the jump frequency is of a similar value to that of the corresponding single SIA and only weakly dependent on the size of the SIA cluster, and (2) for a cluster containing $N$ SIAs, the prefactor of the jump rate exhibited a $1 / N^{\alpha}$ dependence where $\alpha \sim 0.5-0.64 .{ }^{15,18}$ In the work of Osetsky et al. ${ }^{15}$ detailed analysis of atomistic simulations revealed that SIA cluster mobility was largely mediated by individual (but correlated) SIA activity and that with increasing cluster size, such activity became more prevalent in the perimeter region of the cluster. This latter observation was put on firm theoretical grounding via an analysis of the multistring Frenkel-Kontorova model, ${ }^{24}$ which demonstrated that with increasing size, explicit interstitial activity concentrated on the perimeter of the cluster. Moreover, in the limit of large cluster size the perimeter structure would approach that of an edge dislocation and therefore the notion of a prismatic dislocation loop could be formally introduced. Such a viewpoint is also supported by good agreement between atomistic and continuum elasticity descriptions of large SIA clusters. ${ }^{25}$

Experimentally, the use of in situ electron microscopy has allowed for the direct observation of such microstructural dynamics, ${ }^{26-30}$ showing how a population of both mobile and immobile self-interstitial/vacancy clusters diffuse, interact, and can contribute to structural evolution under irradiation. Indeed in the work of Arakawa et al. ${ }^{26}$ a power-law dependence in terms of loop size on mobility was also observed with the jump-rate dependence given by $1 / N^{\alpha}$, where $\alpha \sim 0.8$. More generally, such observations show that the simultaneous production of mobile defects at high irradiation dose-rates results in the evolving microstructures being strongly affected by collective modes involving the correlated migration of defects, formation of rafts of defects, coalescence of defects, and the eventual self-organization and spatial ordering of defects. This indicates that both short and long-range (elastic) interactions between radiation defects play a significant part in the dynamics of microstructural evolution. At higher temperatures, where $\langle 100\rangle$ prismatic loops dominate, such experimental work has also shown the mobility of such loops, ${ }^{27}$ albeit at a significantly reduced rate when compared to $\langle 111\rangle$ prismatic loops.

Direct simulation of such microstructural evolution is not possible using standard molecular dynamics because of the characteristic large length and long time scales. While the above has shown that atomic simulation is able to address the mobility of individual SIA clusters, the simulation of multiple and interacting SIAs becomes impractical due to the computational requirements of system size. Moreover, reaction kinetics (between defect clusters and/or immobile impurity complexes) often involve energy barriers much higher than the thermal energy scale resulting in the relevant diffusion phenomena (and therefore microstructural evolution) occurring at the microseconds to the human time scale - a time regime well beyond that accessible to the molecular-dynamics simulation technique, which is generally limited to the nanosecond time regime. An important example demonstrating this is in the work of Arakawa et al ${ }^{26}$ in which the mobility of interstitial clusters in ion-irradiated bcc Fe was observed to be orders of magnitude less than that seen in simulation suggesting the presence of (unseen) impurity atmospheres with energy barriers much larger than that corresponding to the thermal energy scale. The quantitative resolution of this experimental observation remains an unsolved problem.

Significant progress has been made in the study of microstructural evolution through the use of kinetic Monte Carlo (KMC) methods that allow for the modeling of heterogeneous defect dynamics at time scales extending to the human time scale. ${ }^{31}$ These methods are statistical in nature and can, in the long time limit, correctly describe the temporal dynamics of material evolution. As input, KMC requires kinetic data in the form of migration and reaction energy barriers derived from $a b$ initio and empirical simulations. This demonstrates that the modeling of materials evolution is a truly multiscale modeling methodology. Such an approach has aided in the detailed interpretation of temperature-dependent resistivity recovery data; ${ }^{4}$ a revealing experimental method that is difficult to interpret since the method cannot explicitly distinguish between vacancy and interstitial defect structures and their corresponding reactions.

KMC algorithms for simulating radiation damage effects are generally based on the assumption that defects perform unbiased three-dimensional migration in the material, and that interactions are described as short-range inelastic collisions. The simulations include no treatment of long-range elastic forces or the one-dimensional modes of motion characteristic of prismatic dislocation loops, and therefore cannot presently be used to quantitatively understand the collective defect dynamics observed in in situ electron microscopy.

The Langevin approach provides a powerful alternative to the KMC modeling of defects. It is a method that retains atomic resolution in terms of a defect's position, whilst coarsegraining the atomistic detail of the defect structure keeping only its bare diffusion coefficient and the dimensionality of its mobility. Because of this, the method does not suffer from the time-scale problem normally associated with atomistic simulations, whilst still providing Newtonian-like trajectories whose time scale is set by the corresponding defect's diffusion coefficient. Due to the coarse graining of the atomic lattice, interactions between "Langevin" particles are derived from the appropriate elastic interaction between defects. The omitted atomistic details are accounted for by stochastic differential equations in which a stochastic force is introduced to promote fluctuations within the system whilst a frictional term is added to take into account the dynamics of dissipation associated with the underlying discreteness of the atomic scale. Formally, the Langevin equation is equivalent to a Fokker-Planck equation that governs the probability distribution of the random variables, which in this case are the positions of all defects in the considered system. Such an approach has recently been used to match and explain the real-time dynamics of interacting prismatic dislocation loops in both electron- and ion-irradiated bcc Fe. ${ }^{32}$ 
In the present work the Langevin approach is extended to the modeling of both the internal dynamics and the collective behavior of interstitial (or vacancy) prismatic loops. This is achieved by recognizing that larger loops can be well described by the elasticity of prismatic dislocation loops, which in turn can be described (if the loop is not too large) by the dynamics of an elastic string connected end to end. Section II develops the discretized version of the partial stochastic differential equation for a continuous elastic string within the framework of the Langevin equation. It is shown that the collective diffusion and internal fluctuations of the elastic string are fully and independently described by, respectively, the center-of-mass diffusion coefficient and the elastic string stiffness. In Sec. III, these two microscopic parameters are obtained from finite temperature molecular-dynamics simulations of interstitial and vacancy prismatic loop mobility performed using the magnetic potential for ferromagnetic bcc Fe, ${ }^{20,33}$ from which both the collective diffusion and internal fluctuations of the loop can be directly obtained. In Sec. IV, these results are discussed in terms of existing knowledge of prismatic loops derived from both molecular-dynamics simulation and theory. The Appendix includes a modified derivation of the so-called "drift technique," developed in Refs. 11 and 34, and used here to obtain the converged diffusion coefficients of the atomistically simulated prismatic loops in Sec. III.

\section{DISCRETE ELASTIC STRING MODEL FOR A PRISMATIC LOOP}

The description of a dislocation via a continuum elastic string model and with overdamped dynamics has been used to study the local interaction of a dislocation with pinning sites. Also referred to as the line-tension model, much work in this direction has been performed in the framework of understanding internal friction experiments; see, for example, the early work of Granato and Lücke. ${ }^{35}$ Later work attempted to reconcile such a continuum description with the discreteness of the atomic lattice (via the existence of dislocation kinks), finding an equivalence between the two descriptions when the kink density is high: a regime in which the short-range kinkkink interaction is dominant. ${ }^{36,37}$ It is from this perspective that the present work attempts to describe the internal dynamics of a prismatic dislocation loop. Such an approach has been also taken by Ohsawa and Kuramoto ${ }^{38,39}$ where they studied the migration barrier of an elastic loop in the presence of a Peierls-like energy barrier. Generally, the empirical parameters of the elastic string description are the effective string mass, the viscous drag force, and effective tension per unit length, microscopic parameters whose values depend on the coarse grained features of the discrete lattice. In the present work, a description is developed that makes it possible to obtain these parameters directly from molecular-dynamics simulations of vacancy or interstitial prismatic loops. In the first step of this procedure the differential equation describing the overdamped elastic string is recast in the framework of a stochastic differential equation thereby relating the viscous drag term directly to the mobility properties of the loop via the fluctuation-dissipation theorem, thereby avoiding the need for defining an explicit microscopic mechanism for diffusion.
The elastic string description for a damped dislocation loop under zero applied shear stress conditions obeys the partial differential equation: ${ }^{35}$

$$
\mathcal{M} \frac{\partial^{2} z(l, t)}{\partial t^{2}}+\gamma \frac{\partial z(l, t)}{\partial t}-\mathcal{C} \frac{\partial^{2} z(l, t)}{\partial l^{2}}=0,
$$

where $\mathcal{M}$ is the effective mass per unit length, $\gamma$ is the viscous drag per unit length, and $\mathcal{C}$ is the line tension per unit length. The elastic string is joined end to end via the boundary condition

$$
z(l+L)=z(l) .
$$

To explicitly consider the effects of thermal fluctuations a $\delta$-correlated random force satisfying

$$
\left\langle\zeta(l, t) \zeta\left(l^{\prime}, t^{\prime}\right)\right\rangle=\delta\left(l-l^{\prime}\right) \delta\left(t-t^{\prime}\right),
$$

is introduced giving

$$
\mathcal{M} \frac{\partial^{2} z(l, t)}{\partial t^{2}}+\gamma \frac{\partial z(l, t)}{\partial t}-\mathcal{C} \frac{\partial^{2} z(l, t)}{\partial l^{2}}=f \zeta(l, t) .
$$

Here the parameter $f$ sets the scale of the so-called stochastic force. In the overdamped regime of diffusion, the inertial term becomes negligible and can be omitted resulting in the stochastic partial differential equation:

$$
\gamma \frac{\partial z(l, t)}{\partial t}=\mathcal{C} \frac{\partial^{2} z(l, t)}{\partial l^{2}}+f \zeta(l, t) .
$$

In this form, the Langevin approach is equivalent to a Fokker-Planck equation in which only the spatial degrees of freedom are considered, a form that corresponds to a generalized diffusion equation. The drag (or friction) term may be characterized by $\gamma=k_{b} T / \mathcal{D}$, where $\mathcal{D}$ can be considered as the diffusion constant per unit inverse length, and upon division by the length of the string will give the diffusion coefficient of the entire string. A more convenient form of Eq. (5) is written as

$$
\frac{\partial z(l, t)}{\partial t}=\frac{\mathcal{C D}}{k_{b} T} \frac{\partial^{2} z(l, t)}{\partial l^{2}}+\frac{f \mathcal{D}}{k_{b} T} \zeta(l, t) .
$$

The parameter $f$ may be determined by considering the equation of motion for the center of position (COP) of the elastic string:

$$
\frac{\partial z_{\mathrm{COP}}(t)}{\partial t}=\frac{f \mathcal{D}}{k_{b} T} \frac{1}{L} \int_{0}^{L} d l \zeta(l, t),
$$

where the $\mathrm{COP}$ is defined as

$$
z_{\mathrm{COP}}(t)=\frac{1}{L} \int_{0}^{L} d l z(l, t) .
$$

To obtain Eq. (7), the identity

$$
\left.\int_{0}^{L} d l \frac{\partial^{2} z(l, t)}{\partial l^{2}} \equiv \frac{\partial z(l, t)}{\partial l}\right|_{L}-\left.\frac{\partial z(l, t)}{\partial l}\right|_{0},
$$

which is equal to zero due to the periodic boundary conditions [Eq. (2)], has been used. This last equation simply reflects the fact that the net force on the string is zero.

Equation (7) has the solution

$$
z_{\mathrm{COP}}(\tau)=z_{\mathrm{COP}}(0)+\frac{f \mathcal{D}}{k_{b} T} \frac{1}{L} \int_{0}^{L} d l \int_{0}^{\tau} d t \zeta(l, t) .
$$


Setting the initial COP to be at the origin, $z_{\mathrm{COP}}(0)=0$, the mean-square displacement of $z_{\mathrm{COP}}(\tau)$ is given by

$$
\begin{aligned}
\left\langle\left|z_{\mathrm{COP}}(\tau)\right|^{2}\right\rangle= & \left(\frac{f \mathcal{D}}{k_{b} T L}\right)^{2} \int_{0}^{L} d l \int_{0}^{L} d l^{\prime} \\
& \times \int_{0}^{\tau} d t \int_{0}^{\tau} d t^{\prime}\left\langle\zeta(l, t) \zeta\left(l^{\prime}, t^{\prime}\right)\right\rangle \\
= & \left(\frac{f \mathcal{D}}{k_{b} T L}\right)^{2} L \tau \equiv 2 D_{\mathrm{COP}} \tau
\end{aligned}
$$

By definition, $D_{\mathrm{COP}}$ equals the diffusion coefficient of the entire loop and is equal to $\mathcal{D} / L$. Thus, by requiring that the COP exhibits Brownian motion, characterized by a diffusion coefficient equal to $\mathcal{D} / L, f$ is defined and results in Eq. (6) becoming

$$
\frac{\partial z(l, t)}{\partial t}=\frac{\mathcal{C D}}{k_{b} T} \frac{\partial^{2} z(l, t)}{\partial l^{2}}+\sqrt{2 \mathcal{D}} \zeta(l, t) .
$$

This constitutes an example of the application of the fluctuation-dissipation theorem.

With the goal being the development of a numerical simulation strategy, Eq. (13) is now discretized by replacing the second-order differential term with a finite-difference representation defined in terms of the segment length $\Delta L$,

$$
\begin{aligned}
\frac{\partial z(l, t)}{\partial t}= & \frac{\mathcal{C D}}{k_{b} T}\left[\frac{z(l-\Delta L, t)-2 z(l, t)+z(l+\Delta L, t)}{(\Delta L)^{2}}\right] \\
& +\sqrt{2 \mathcal{D}} \zeta(l, t),
\end{aligned}
$$

and averaging within all such segment lengths,

$$
\begin{aligned}
\frac{\partial z_{n}(t)}{\partial t}= & \frac{\mathcal{C D}}{k_{b} T(\Delta L)^{2}}\left[z_{n-1}(t)-2 z_{n}(t)+z_{n+1}(t)\right] \\
& +\sqrt{2 \mathcal{D}} \zeta_{n}(t) .
\end{aligned}
$$

Here

$$
z_{n}(t)=\frac{1}{\Delta L} \int_{l_{n}-\Delta L / 2}^{l_{n}+\Delta L / 2} d l z(l, t)
$$

and

$$
\zeta_{n}(t)=\frac{1}{\Delta L} \int_{l_{n}-\Delta L / 2}^{l_{n}+\Delta L / 2} d l \zeta(l, t) .
$$

In the above $l_{n+1}-l_{n}=\Delta L$ and $n$ is chosen to range from zero to $L / \Delta L-1=N-1$. Using Eq. (3), the discrete form of the $\delta$-correlated spatiotemporal noise evaluates to

$$
\begin{aligned}
\left\langle\zeta_{n}(t) \zeta_{n^{\prime}}\left(t^{\prime}\right)\right\rangle & =\int_{l_{n}-\Delta L / 2}^{l_{n}+\Delta L / 2} d l \int_{l_{n^{\prime}}-\Delta L / 2}^{l_{n^{\prime}}+\Delta L / 2} d l^{\prime} \delta\left(l-l^{\prime}\right) \delta\left(t-t^{\prime}\right) \\
& =\frac{1}{\Delta L} \delta_{n, n^{\prime}} \delta\left(t-t^{\prime}\right) .
\end{aligned}
$$

The final form of the discrete differential equation is then given by

$$
\begin{aligned}
\frac{\partial z_{n}(t)}{\partial t}= & \frac{\mathcal{C D}}{k_{b} T(\Delta L)^{2}}\left[z_{n-1}(t)-2 z_{n}(t)+z_{n+1}(t)\right] \\
& +\sqrt{2 \frac{\mathcal{D}}{\Delta L}} \zeta_{n}(t)
\end{aligned}
$$

with

$$
\left\langle\zeta_{n}(t) \zeta_{n^{\prime}}\left(t^{\prime}\right)\right\rangle=\delta_{n, n^{\prime}} \delta\left(t-t^{\prime}\right) .
$$

These equations may be readily transformed to a series of uncoupled Langevin equations via the discrete Fourier transform

$$
\bar{z}_{k}(t)=\frac{1}{N} \sum_{n=0}^{N-1} z_{n}(t) \exp \left(\frac{i 2 \pi n k}{N}\right)
$$

and its inverse transform

$$
z_{n}(t)=\sum_{k=0}^{N-1} \bar{z}_{k}(t) \exp \left(-\frac{i 2 \pi n k}{N}\right) .
$$

It is noted that

$$
\bar{z}_{0}(t)=\frac{1}{N} \sum_{n=0}^{N-1} z_{n}(t)=z_{\mathrm{COP}}(t),
$$

which is the COP for the elastic string. The resulting $N$ independent Fourier transformed Langevin equations are

$$
\frac{d \bar{z}_{k}(t)}{d t}=-\lambda_{k} \bar{z}_{k}(t)+\sqrt{2 \frac{\mathcal{D}}{\Delta L}} \bar{\zeta}_{k}(t)
$$

where

$$
\lambda_{k}=\frac{2 \mathcal{C D}}{k_{b} T \Delta L^{2}}\left[1-\cos \left(\frac{2 \pi k}{N}\right)\right]
$$

and

$$
\bar{\zeta}_{k}(t)=\frac{1}{N} \sum_{n=0}^{N-1} \zeta_{n}(t) \exp \left(\frac{i 2 \pi n k}{N}\right) .
$$

In the above definitions, both $z_{n}(t)$ and $\zeta_{n}(t)$ are real and therefore their Fourier coefficients satisfy the following condition: (i) The $k=0$ and $k=N / 2$ (if $N$ is even) Fourier coefficients are real. (ii) The $k$ th coefficient equals the conjugate of the $(N-k)$ th coefficient for $k=1, N / 2-1$ if $N$ is even, and for $k=1,(N-1) / 2-1$ if $N$ is odd.

To ensure that this is the case for $\bar{\zeta}_{k}(t)$, the $N$ independent Fourier-space random forces are assigned to the real numbers $\bar{\zeta}_{k=0}(t)$ [and $\bar{\zeta}_{k=N / 2}(t)$ if $N$ is even] and the real and imaginary parts of the complex numbers $\bar{\zeta}_{k}(t)$, where $k=$ $1, N / 2-1$ if $N$ is even, and $k=1,(N-1) / 2-1$ if $N$ is odd. Doing so results in the following $\delta$-correlated temporal correlation functions for the Fourier transformed random forces:

$$
\left\langle\bar{\zeta}_{k}(t)\left[\bar{\zeta}_{k^{\prime}}\left(t^{\prime}\right)\right]^{\dagger}\right\rangle=\frac{1}{N} \delta_{k, k^{\prime}} \delta\left(t-t^{\prime}\right) .
$$

For $k=0, \lambda_{k}=0$ and Eq. (24) reduces to

$$
\frac{d \bar{z}_{0}(t)}{d t}=\sqrt{2 \frac{\mathcal{D}}{\Delta L}} \bar{\zeta}_{0}(t),
$$

which has the solution

$$
\bar{z}_{0}(\tau)=\bar{z}_{0}(0)+\sqrt{2 \frac{\mathcal{D}}{\Delta L}} \int_{0}^{\tau} d t \bar{\zeta}_{0}(t),
$$


resulting in [when setting $\bar{z}_{k}(0)$ to zero]

$$
\begin{aligned}
\left\langle\left|\bar{z}_{0}(\tau)\right|^{2}\right\rangle & =2 \frac{\mathcal{D}}{\Delta L} \int_{0}^{\tau} d t \int_{0}^{\tau} d t^{\prime}\left\langle\bar{\zeta}_{0}(t)\left[\bar{\zeta}_{0}\left(t^{\prime}\right)\right]^{\dagger}\right\rangle \\
& =2 \frac{\mathcal{D}}{\Delta L} \frac{\tau}{N}=2 \frac{\mathcal{D}}{L} \tau,
\end{aligned}
$$

which is in agreement with Eq. (12). This result does not depend on the choice of the number of nodes $N$.

For $k \neq 0$, the general solution to Eq. (24) takes the form

$\bar{z}_{k}(\tau)=\exp \left(-\lambda_{k} \tau\right)\left[\bar{z}_{k}(0)+\sqrt{2 \frac{\mathcal{D}}{\Delta L}} \int_{0}^{\tau} d t \exp \left(\lambda_{k} t\right) \bar{\zeta}_{k}(t)\right]$

leading to

$$
\begin{aligned}
\left\langle\bar{z}_{k}(\tau)\left[\bar{z}_{k^{\prime}}(\tau)\right]^{\dagger}\right\rangle= & \exp \left[-\left(\lambda_{k}+\lambda_{k^{\prime}}\right) \tau\right]\left[\bar{z}_{k}(0)\left[\bar{z}_{k^{\prime}}(0)\right]^{\dagger}\right. \\
& +2 \frac{\mathcal{D}}{\Delta L} \int_{0}^{\tau} d t \int_{0}^{\tau} d t^{\prime} \\
& \left.\times \exp \left(\lambda_{k} t\right) \exp \left(\lambda_{k^{\prime}} t^{\prime}\right)\left\langle\bar{\zeta}_{k}(t)\left[\bar{\zeta}_{k^{\prime}}\left(t^{\prime}\right)\right]^{\dagger}\right\rangle\right] \\
= & \exp \left[-\left(\lambda_{k}+\lambda_{k^{\prime}}\right) \tau\right]\left[\bar{z}_{k}(0)\left[\bar{z}_{k^{\prime}}(0)\right]^{\dagger}\right. \\
& \left.+\frac{2 \mathcal{D}}{\Delta L N} \int_{0}^{\tau} d t \exp \left(2 \lambda_{k} t\right) \delta_{k, k^{\prime}}\right] .
\end{aligned}
$$

If $k \neq k^{\prime}$ then

$$
\begin{aligned}
& \left\langle\bar{z}_{k}(\tau)\left[\bar{z}_{k^{\prime}}(\tau)\right]^{\dagger}\right\rangle \\
& \quad=\exp \left[-\left(\lambda_{k}+\lambda_{k^{\prime}}\right) \tau\right] \bar{z}_{k}(0)\left[\bar{z}_{k^{\prime}}(0)\right]^{\dagger} \rightarrow 0
\end{aligned}
$$

in the long time limit $(\tau \rightarrow \infty)$, whereas if $k=k^{\prime}$,

$$
\begin{aligned}
\left\langle\left|\bar{z}_{k}(\tau)\right|^{2}\right\rangle & =\exp \left(-2 \lambda_{k} \tau\right)\left[\left|\bar{z}_{k}(0)\right|^{2}+\frac{2 \mathcal{D}}{\Delta L N} \frac{\exp \left(2 \lambda_{k} \tau\right)-1}{2 \lambda_{k}}\right] \\
& \rightarrow \frac{\mathcal{D}}{\Delta L N \lambda_{k}} .
\end{aligned}
$$
by

The instantaneous spatial correlation function is then given

$$
\begin{aligned}
& \left\langle z_{n}(\tau)\left[z_{n^{\prime}}(\tau)\right]^{\dagger}\right\rangle \\
& =\sum_{k, k^{\prime}=0}^{N-1}\left\langle\bar{z}_{k}(\tau)\left[\bar{z}_{k^{\prime}}(\tau)\right]^{\dagger}\right\rangle \exp \left(-\frac{i 2 \pi n k}{N}\right) \exp \left(\frac{i 2 \pi n^{\prime} k^{\prime}}{N}\right) \\
& =\frac{2 \mathcal{D} \tau}{\Delta L N}+\sum_{k=1}^{N-1} \frac{\mathcal{D}}{\Delta L N \lambda_{k}} \exp \left(-\frac{i 2 \pi\left(n-n^{\prime}\right) k}{N}\right)
\end{aligned}
$$

where in the last step the results contained in Eqs. (30), (33), and (34) have been used. It is noted that in the very last term of Eq. (35) the summation in $k$ does not include the $k=0$ term. Using Eq. (25) gives

$$
\left\langle z_{n}(\tau)\left[z_{n^{\prime}}(\tau)\right]^{\dagger}\right\rangle=\frac{2 \mathcal{D} \tau}{\Delta L N}+\frac{k_{b} T \Delta L}{2 \mathcal{C}} G\left(n, n^{\prime}\right),
$$

where

$$
G\left(n, n^{\prime}\right)=\frac{1}{N} \sum_{k=1}^{N-1} \frac{\exp \left(-\frac{i 2 \pi\left(n-n^{\prime}\right) k}{N}\right)}{1-\cos \left(\frac{2 \pi k}{N}\right)} .
$$

$G\left(n, n^{\prime}\right)$ evaluates to a real function for all values of $n$ and $n^{\prime}$, and has a form similar to the static $(\omega=0)$ phonon spatial Green's function for a one-dimensional peroidic Harmonic chain.

Equation (36) allows for a direct calculation of the meansquare displacement of the COP of the loop,

$$
z_{\mathrm{COP}}(\tau)=\frac{1}{N} \sum_{n=1}^{N} z_{n}(\tau)
$$

That is,

$$
\begin{aligned}
\left\langle\left|z_{\mathrm{COP}}(\tau)\right|^{2}\right\rangle & =\frac{1}{N^{2}} \sum_{n, n^{\prime}=1}^{N}\left\langle z_{n}(\tau)\left[z_{n^{\prime}}(\tau)\right]^{\dagger}\right\rangle \\
& =\frac{1}{N^{2}} \sum_{n, n^{\prime}=1}^{N}\left[\frac{2 \mathcal{D} \tau}{\Delta L N}+\frac{k_{b} T \Delta L}{2 \mathcal{C}} G\left(n, n^{\prime}\right)\right] \\
& =\frac{2 \mathcal{D} \tau}{\Delta L N}=\frac{2 \mathcal{D} \tau}{L}
\end{aligned}
$$

where in the last step, the sum rule,

$$
\sum_{n=0}^{N} G\left(n, n^{\prime}\right)=\sum_{n^{\prime}=0}^{N} G\left(n, n^{\prime}\right)=0
$$

has been exploited. This identity may be easily established by using Eq. (37) and the fact that each summation in spatial index yields a Kronecker $\delta$ in $k$ that is equal to unity only for $k=0$, a term that is not included in the summation of Eq. (37).

As expected, Eq. (39) gives an identical result to that of Eqs. (12) and (30), or that derived directly from Eq. (19). In a similar way, the mean-square fluctuation of the nodal positions relative to the COP can be evaluated giving, for all $n$,

$$
\begin{aligned}
& \left\langle\left|z_{n}(\tau)-z_{\mathrm{COP}}(\tau)\right|^{2}\right\rangle \\
& \quad=\left\langle\left|z_{n}(\tau)\right|^{2}\right\rangle-2\left\langle z_{n}(\tau)\left[z_{\mathrm{COP}}(\tau)\right]^{\dagger}\right\rangle+\left\langle\left|z_{\mathrm{COP}}(\tau)\right|^{2}\right\rangle \\
& \quad=\frac{k_{b} T \Delta L}{2 \mathcal{C}} G(n, n) .
\end{aligned}
$$

$G\left(n, n^{\prime}\right)$ may be readily numerically evaluated for a given $N-$ it is well behaved due to the omission of the $k=0$ term. Figure 1 plots $G(n, n) / N$ as a function of $N$ and it is seen that for $N>5$ it is well approximated by the constant $1 / 6$. Also included in this figure are the results of dynamical (interacting $N$-body) Langevin simulations for reasonable (but arbitrarily) chosen values of $\mathcal{D}$ and $\mathcal{C}$ - see Sec. III C.

Thus Eq. (41) gives the central result

$$
\left\langle\left|z_{n}(\tau)-z_{\mathrm{COP}}(\tau)\right|^{2}\right\rangle=\left\langle|\delta z|^{2}\right\rangle=\frac{k_{b} T \Delta L N}{12 \mathcal{C}}=\frac{k_{b} T L}{12 \mathcal{C}},
$$

showing that the magnitude of the fluctuations scales linearly with the perimeter of the loop and the temperature of the system. As with the COP diffusion coefficient, this result does not depend on the choice of $N$. Thus a prescription to determine the (bare) microscopic parameters of the loop becomes evident. By matching the COP diffusion coefficient with the known diffusion coefficient of a similarly sized loop, $\mathcal{D}$ can be determined, and by matching the scale of the fluctuations within the loop with a known value, $\mathcal{C}$ can be obtained. Such microscopic data are directly obtainable 


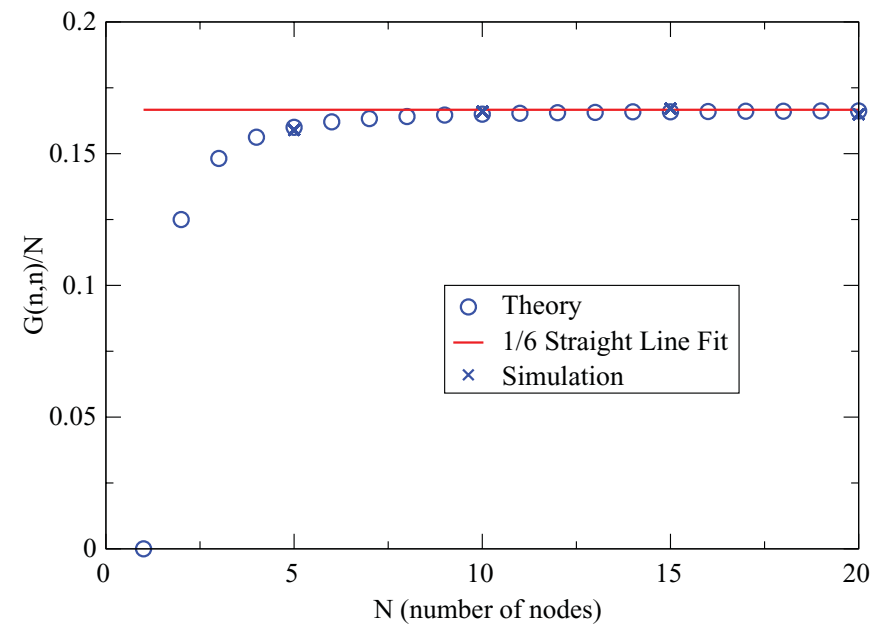

FIG. 1. (Color online) A plot of $G(n, n) / N$ as a function of $N$ where the red line represents $1 / 6$. The stars represent values of $G(n, n) / N$ taken directly from fully dynamical Langevin simulations.

from molecular-dynamics simulations, and thus a multiscale modeling strategy naturally emerges to model loops at time and spatial scales well beyond that of atomistic simulation methods.

\section{DETERMINING $\mathcal{C}$ AND $\mathcal{D}$ FOR $\alpha$-FE FROM MOLECULAR-DYNAMICS SIMULATIONS}

\section{A. Interstitial prismatic loops}

To obtain estimates of the COP diffusion coefficient $\mathcal{D} / L$ and the line tension $\mathcal{C} / L$ a $\langle 111\rangle$ interstitial prismatic loop is created by inserting a $\langle 111\rangle$ platelet of atoms of hexagonal shape into an otherwise perfect bcc lattice of approximately cubic shape, with the coordinate axes orientated along $x=$

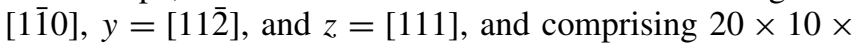
40 unit cells of size $a(\sqrt{2} \times \sqrt{6} \times \sqrt{3} / 2)$, where $a$ is the lattice constant (48000 atoms in total). The number of atoms in the platelet is 61 , corresponding to an area of $\approx 234.5 \AA^{2}$ and a perimeter of $L \approx 57 \AA$. This initial configuration is relaxed to a local energy minimum using molecular statics. To describe the $\mathrm{Fe}-\mathrm{Fe}$ interaction, the empirical magnetic potential is employed. ${ }^{20,33}$ Figure 2(a) displays the resulting structure, showing only those atoms with a cohesive energy $0.15 \mathrm{eV}$ greater than the average local cohesive energy of the sample. To investigate the finite temperature dynamics of this prismatic loop, a series of eight finite temperature moleculardynamics simulations were performed for the temperature range $100-800 \mathrm{~K}$ in increments of $100 \mathrm{~K}$. The simulations were performed under zero hydrostatic pressure conditions using the Parrinello-Rahman barostat method. To obtain sufficient statistics, each simulation was run for $2 \mathrm{~ns}$ and every $100 \mathrm{fs}$ the atomic positions were stored for those atoms with a cohesive energy $0.15 \mathrm{eV}$ greater than the instantaneous average local cohesive energy of the entire sample.

Closer inspection of Fig. 2(a) reveals that at $0 \mathrm{~K}$ the core region consists of a perimeter of high-energy atoms aligned along the $\langle 111\rangle$ axis. This scenario is approximately maintained for the temperature range considered [see Fig. 2(b) for the case of $400 \mathrm{~K}$ ], although the core region is now somewhat more irregular due to increasing noise in the local cohesive energy arising from thermal vibrations. Indeed, many atoms not at all associated with the dislocation loop now survive the energy filtering process and correspond to random high-energy fluctuations occurring within the bulk lattice. Thus with rising temperature, the data become increasingly noisy and further processing is required. To do such postprocessing of the atomic positions, clusters of atoms along a $\langle 111\rangle$ direction are identified according to a nearest-neighbor criterion, and all clusters containing less than four atoms are removed. Also, any remaining cluster that is not located within a chosen distance (a factor of its known radius) to the loop is removed. The positions of the atoms in the remaining clusters

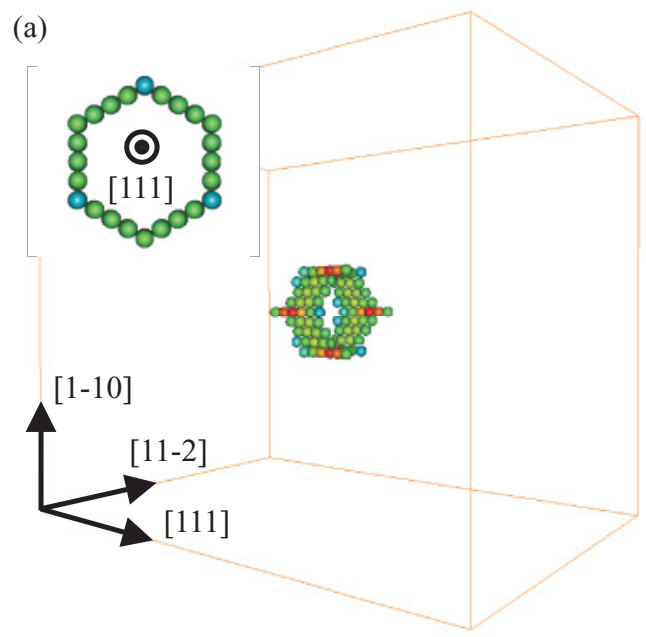

(b)

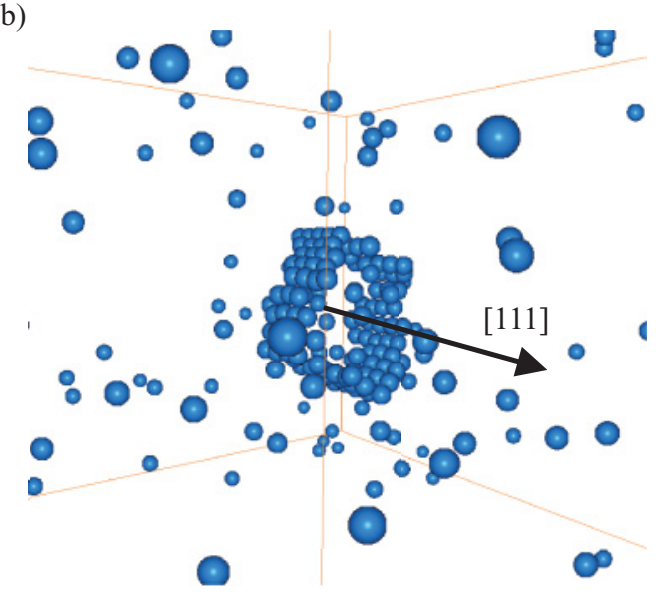

FIG. 2. (Color online) (a) The 0-K relaxed starting configuration for the interstitial prismatic loop containing 61 self-interstitial atoms. Only high-energy atoms are shown and are colored according to their local cohesive energy (red, the most negative, and light blue, the least negative). The displayed bounding box represents the cell size in which the simulations were performed. The inset shows the prismatic loop with the viewing direction along the $\langle 111\rangle$ Burgers vector direction. (b) Instantaneous three-dimensional snapshot of the prismatic loop at a temperature of $400 \mathrm{~K}$; only high-energy atoms are shown. 

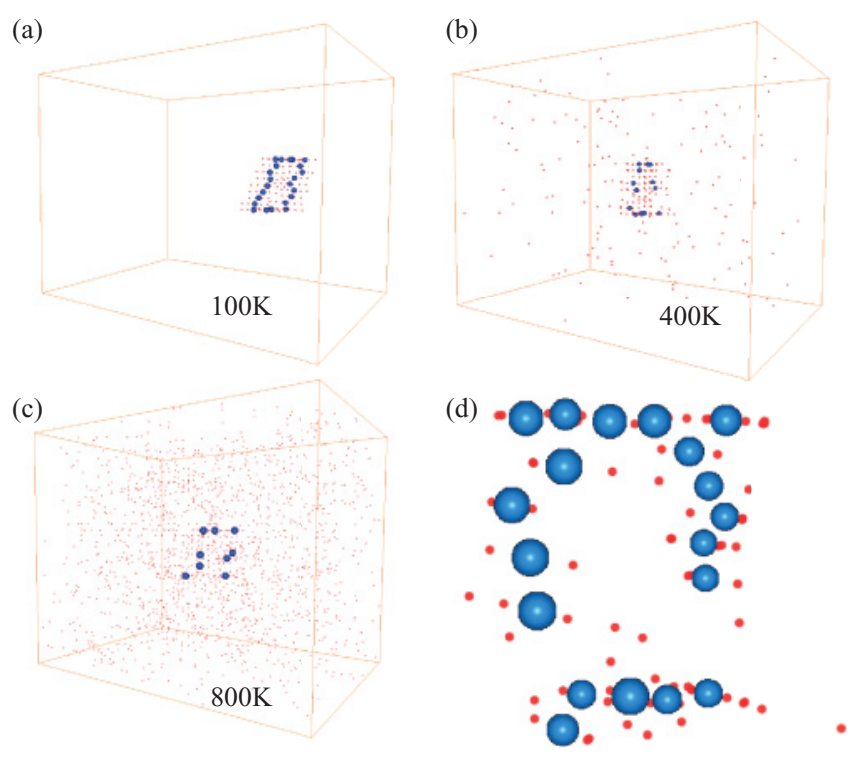

FIG. 3. (Color online) Examples of loop postprocessing at temperatures (a) $100 \mathrm{~K}$, (b) $400 \mathrm{~K}$, and (c) $800 \mathrm{~K}$. The small red circles represent the filtered high-energy atoms and the blue balls represent the resulting configuration of the loop using the postprocessing procedure outlined in the text. (d) Closeup of a loop at $400 \mathrm{~K}$ displaying its identified $\langle 111\rangle$ strings (points) and their respective COPs (balls) that are used to characterize the loop's instantaneous configuration.

are then averaged within each cluster to finally obtain the current configuration of the loop. Figures 3(a)-3(c) display the atomic configurations arising from the molecular-dynamics simulations for the temperatures 100,400 , and $800 \mathrm{~K}$, showing that with increasing temperature the dislocation loop position and shape become more difficult to identify. Figure 3(d) displays a closeup of a loop via its identified $\langle 111\rangle$ strings.

The resulting coordinates of the loop are then used to calculate the COP of the loop $z_{\mathrm{COP}}$ and the mean-square fluctuations in the $\langle 111\rangle$ direction:

$$
\frac{1}{N} \sum_{i}^{N}\left(z_{i}-z_{\mathrm{COP}}\right)^{2} .
$$

Here the summation is over the $N$ identified $\langle 111\rangle$ strings constituting the loop at a given instant (or interval) of time. $N$ will vary from configuration to configuration (especially at the higher temperatures), but will generally have a maximum value of 24 [see Fig. 3(a)]. For each temperature, Eq. (43) is averaged over all the $(\approx 20000)$ configurations obtained during the 2-ns molecular-dynamics simulation. To obtain the diffusion coefficient from the time series of $z_{\mathrm{COP}}$ at each temperature, the mean-square displacement is calculated for a selected range of time intervals using the "drift correction" technique developed in previous work. ${ }^{11,34}$ The as-published method, which ensures that the mean position of a diffusing particle remains zero when sampling a trajectory of finite length, was found to consistently overestimate the diffusion coefficient by a factor of 3 when compared to more traditional methods. The Appendix details the origin of this difference, shedding further light into the statistics of particle trajectories.
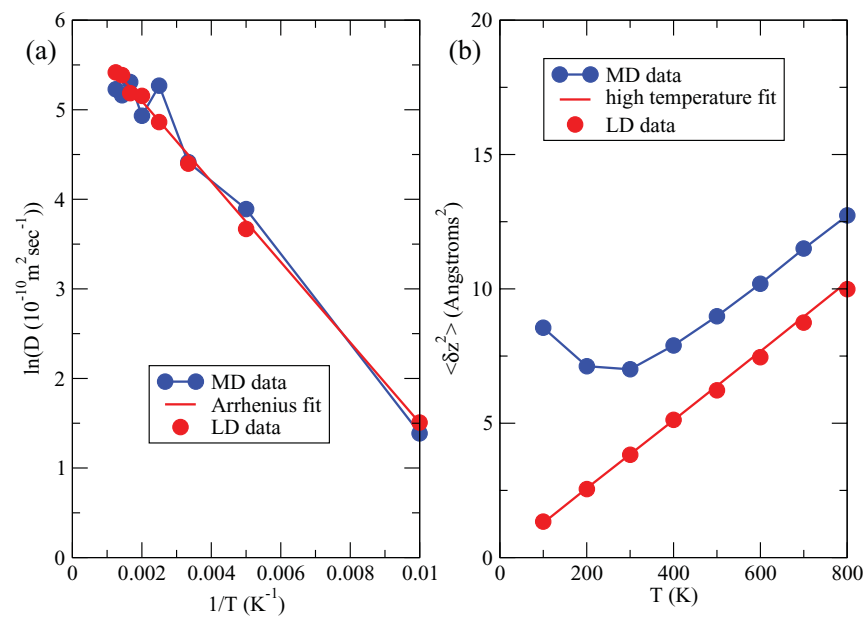

FIG. 4. (Color online) Molecular-dynamics data from an interstitial prismatic loop where (a) is an Arrhenius plot of the measured diffusion coefficients as a function of inverse temperature and (b) is the mean-square fluctuations along the direction of the $\langle 111\rangle$ Burgers vector as a function of temperature. In (a), the red line represents an Arrhenius fit and (b) represents a linear fit to the high-temperature mean-square-fluctuation data, adjusted such that it extrapolates to the origin. The red data points represent the corresponding quantities derived directly from the Langevin dynamics loop trajectories.

Figure 4(a) displays the natural logarithm of the resulting diffusion coefficients as a function of inverse temperature. Inspection of this figure reveals a temperature dependence that is not so well represented by simple Arrhenius behavior. Much better agreement with Arrhenius behavior for bcc $\mathrm{Fe}$ is seen in the work of Osetsky et al. ${ }^{15}$ and more recently in Refs. 16 and 17. This is quite different from the strongly non-Arrhenius behavior seen in molecular-dynamics simulations of interstitial cluster mobility in vanadium. ${ }^{18}$ Indeed in the work on vanadium, the authors comment on this, stating that this might be due to the relatively narrow temperature regime that can be studied in $\mathrm{Fe}$ compared to that of vanadium. With the understanding that an Arrhenius description of cluster diffusion is most probably an oversimplification of the actual diffusion mechanism, the prefactor and migration energy barrier are calculated from Fig. 4(a) giving $D_{0}=$ $392.69 \times 10^{-10} \mathrm{~m}^{2} \mathrm{sec}^{-1}$ and $E=0.0386 \mathrm{eV}$, the latter value being quite comparable to that expected for a $\langle 111\rangle$ crowdion in bcc Fe. As in previous work, this suggests that the microscopic mechanism, which facilitates loop diffusion, is in part related to the mobility of the $\langle 111\rangle$ SIA crowdion, a defect that on its own decays to the ground-state $\langle 110\rangle$ SIA defect structure, but in a many-SIA loop configuration generates the shortest Burgers magnitude corresponding to the minimum-energy structure predicted by dislocation theory.

Figure 4(b) displays the resulting mean-square fluctuations of the loop [as defined by Eq. (43)] as functions of temperature using the postprocessed data. In the high-temperature regime, the fluctuations depend linearly on temperature as predicted by Eq. (42). However, with decreasing temperature a strong deviation away from linearity is apparent-indeed the mean-square fluctuations appear to increase with decreasing temperature when below $300 \mathrm{~K}$. This behavior may be rationalized by 

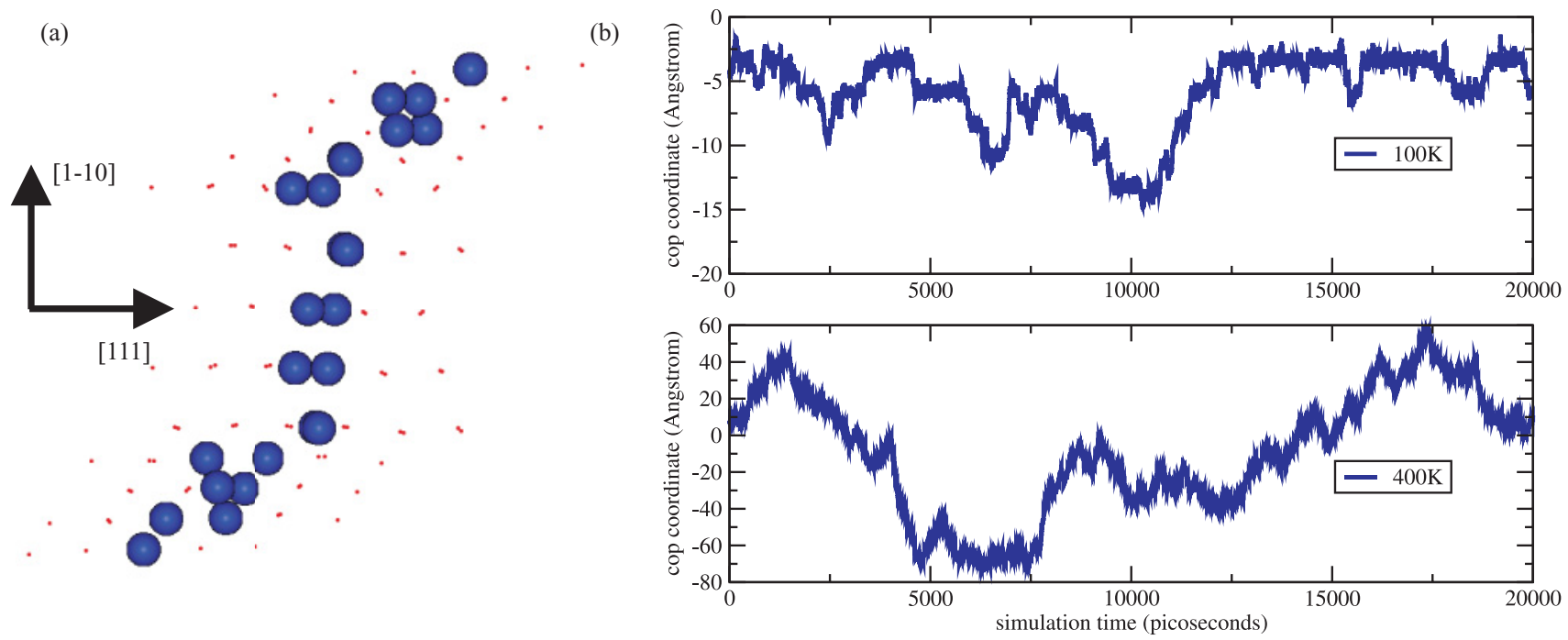

FIG. 5. (Color online) (a) Typical loop configuration consisting of 51 SIAs at $100 \mathrm{~K}$ (with viewing direction along the [11 $\overline{2}]$ axis) with a habit plane not perpendicular to the [111] Burgers vector direction. (b) Measured loop's COP as a function of simulation time at 100 and $400 \mathrm{~K}$.

inspecting the typical loop configurations at these low temperatures. Figure 5(a) displays such a configuration at $100 \mathrm{~K}$ and it is seen that the corresponding habit plane of the loop is not in the [111] direction associated with the loop's Burgers vector. Such configurations can last many nanoseconds suggesting that the low-temperature ground state of the prismatic loop is not that of the relaxed $0-\mathrm{K}$ structure of Fig. 2 [where the habit plane is (111)], but rather that shown in Fig. 5(a) with its normal along the [110] direction, an observation that has been also noticed by Wirth $e t$ al. ${ }^{6}$ Under these circumstances, the application of Fig. 4(b) is somewhat nonsensical, since a static non-\{111\} habit plane configuration will yield a large value for the instantaneous fluctuation. That the mean-squared fluctuation increases in Fig. 5(b) with decreasing temperature is thus simply a result of the prismatic loop spending much of its time in its non- $\{111\}$ habit plane configuration. Longer simulation times at these lower temperatures should result in a less nonlinear temperature dependence since the dislocation loop will have time to "flip" its habit plane to one of the other two equivalent $\langle 110\rangle$ habit planes. Using the values of the mean-squared fluctuations at 700 and $800 \mathrm{~K}$, an estimate of the gradient yields $0.012 \AA^{2} \mathrm{~K}^{-1}$, the necessary microscopic quantity to determine the line tension coefficient $\mathcal{C}$.

From the above analysis, the simple elastic string model developed in the previous section becomes applicable at temperatures above $300 \mathrm{~K}$. Below this temperature, the continuum approximation inherent to the elastic string description breaks down and the discreteness of the atomic structure becomes apparent with strong deviations away from the linear temperature dependence of the mean-square fluctuations predicted by Eq. (42). Additionally, it is noted that even the high-temperature linear region of Fig. 4(b) does not intersect the origin when extrapolated to $T=0 \mathrm{~K}$, indicating a inherent discreteness at the atomic scale, where internal fluctuations cannot be less than typical interatomic distances. The emergence of atomic discreteness at the lower temperatures also manifests itself in the nature of the diffusion. Figure 5(b) shows that at $100 \mathrm{~K}$, the path the loop takes is more discrete than say at $400 \mathrm{~K}$, which is more typical of the self-similar Brownian motion that the Langevin technique can model.

\section{B. Vacancy prismatic loops}

The vacancy prismatic dislocation loop was prepared in a way inverse to that of the interstitial case, where, rather than adding a $\langle 111\rangle$ platelet of 61 atoms, an entire plane of atoms was added except within the radius of the required loop. After relaxation this created the required "collapsed" vacancy dislocation loop. ${ }^{21}$ The details of the simulation cell and the raw data collection methods were otherwise identical to that of the interstitial loop simulations. Visual inspection of the highenergy atomic configurations revealed, at all temperatures, a much more ill-defined dislocation loop resulting in the interstitial loop postprocessing procedure of locating clusters of individual $\langle 111\rangle$ strings not being applicable. The more difficult nature of identifying the actual location and instantaneous configuration of the vacancy prismatic loop is possibly due to incomplete relaxation of the loop during its initial creation, or more generally due to the molecular-dynamics observation that the vacancy prismatic loop structure is in fact a metastable structure, having a higher formation energy than a comparable spherical void. $^{21}$

It was found that a more reliable approach was to, instead, identify small unit clusters of two to four atoms that existed over a short temporal period as belonging to the cluster, and using those to construct the instantaneous position of the loop. Figure 6 displays the resulting diffusion coefficient and mean-square fluctuations along the $\langle 111\rangle$ Burgers vector of the loop. The diffusion coefficient was found to be independent of the choice of the unit-cluster size, whereas the mean-square fluctuations were found to depend on it rather strongly. Despite this, the mean-square fluctuations as a function of increasing temperature show the same qualitative behavior for all choices of unit-cluster size where there is a deviation away from linearity at lower temperatures. At the lowest temperature, 

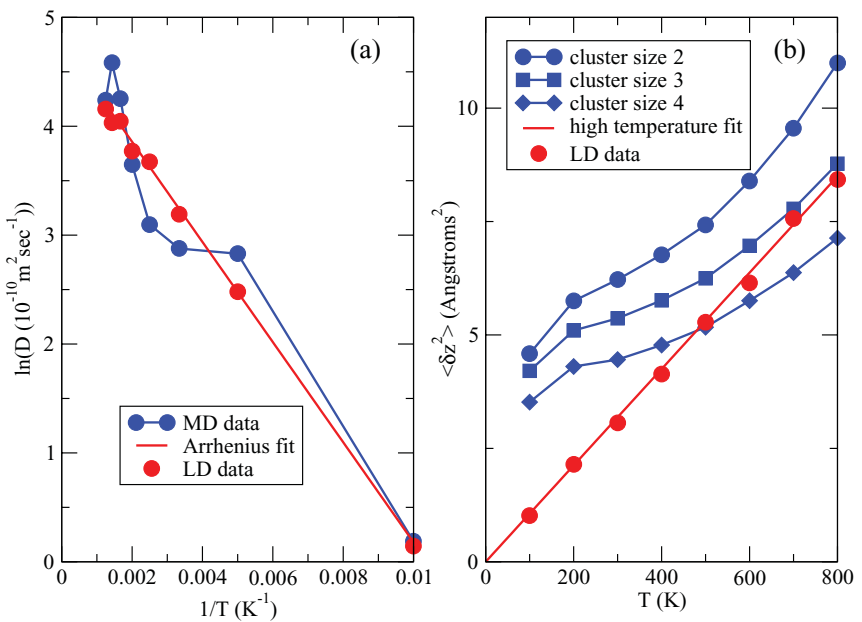

FIG. 6. (Color online) Molecular-dynamics data from a vacancy prismatic loop where (a) is an Arrhenius plot of the measured diffusion coefficients as a function of inverse temperature and (b) is the mean-square fluctuations along the direction of the $\langle 111\rangle$ Burgers vector as a function of temperature, using three different unit-cluster sizes (see text). In (a), the red line represents an Arrhenius fit and (b) represents a linear fit to the high-temperature mean-square-fluctuation data, adjusted such that it extrapolates to the origin. The red data points represent the corresponding quantities derived directly from the Langevin dynamics loop trajectories.

there exists a distinct change in behavior, corresponding to a drop in internal fluctuations, which Fig. 7 reveals to be due to a change in how the vacancy cluster moves. At this lowest temperature, the cluster remains stationary with intermediate jumps to neighboring positions, whereas at higher temperatures, a more self-similar behavior (as in the case of the interstitial clusters) is apparent. Visual inspection of the vacancy cluster configuration at this low temperature showed no deviation of the habit plane from a normal defined by its Burgers vector. Indeed, even at higher temperatures, the habit

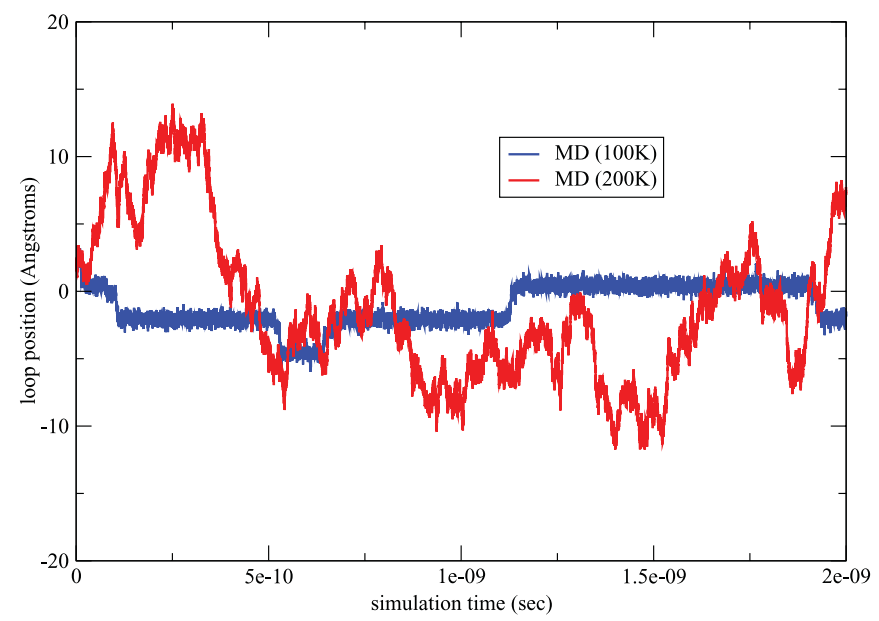

FIG. 7. (Color online) Trajectories of a vacancy prismatic dislocation loop at 100 and $200 \mathrm{~K}$ obtained using molecular dynamics. At the temperature of $100 \mathrm{~K}$, there is a distinctly different behavior where the vacancy stays stationary jumping intermittently to a nearby position, whereas at a temperature of $200 \mathrm{~K}$ (and above) the trajectories are less discrete and visually more self-similar. plane of a vacancy dislocation loop is much more stable than that of the interstitial case due to the absence of an alternative favourable configuration of two adjacent vacancies-in the interstitial case two crowdions tend to offset from one-another, but this does not happen with vacancies.

Figure 6(a) shows that the temperature dependence of the diffusion coefficient is somewhat more scattered than that of the interstitial data [Fig. 4(a)]. This might, however, be due to the more difficult nature of identifying its actual position and, in the case of the 100-K data, insufficient statistics. Despite the scattered nature of the data, an Arrhenius fit gave $D_{0}=$ $118.69 \times 10^{-10} \mathrm{~m}^{2} \mathrm{sec}^{-1}$ and $E=0.0396 \mathrm{eV}$. Interestingly, the diffusion coefficient of the vacancy prismatic loop is not so different from its interstitial counterpart, demonstrating that vacancy transport via such prismatic loops is comparable to the interstitial mechanism, if they are able to be formed. Indeed the Arrenhius energy barrier is comparable to that of the interstitial prismatic loop, whereas the prefactor is three to four times smaller. Recent in situ and ex situ TEM investigations of loop formation in $\mathrm{Fe}$ and $\mathrm{Fe}-\mathrm{Cr}$ alloys suggested that a certain percentage of the observed loops were of the vacancy type $^{28}$ although the exact mechanism for the creation of these metastable defects remains unclear. Fast one-dimensional migration of vacancy clusters in fcc metals has also been observed using similar in situ methods. ${ }^{30}$ The fact that vacancy loops are generally only observed in experiments where the dose rate is high enough to guarantee a large amount of cascade overlap (in the case of heavy-ion irradiation) suggests that they can only form when the local vacancy density is sufficiently high, and even then the probability is still low, with the formation of voids dominating. ${ }^{29}$

\section{Simulation of elastic loop using Langevin dynamics}

To make the developed Langevin formalism more easily transferable to a typical molecular-dynamics code, Eq. (19) is written in the more general form,

$$
\frac{\partial z_{n}(t)}{\partial t}=-\frac{1}{k_{b} T} \frac{\mathcal{D}}{\Delta L} \frac{\partial U}{\partial z_{n}(t)}+\sqrt{2 \frac{\mathcal{D}}{\Delta L}} \zeta_{n}(t),
$$

where

$$
\begin{aligned}
U & =U\left(z_{1}(t), z_{2}(t), \ldots, z_{N}(t)\right) \\
& =\sum_{n=1}^{N} \frac{1}{2} \frac{\mathcal{C}}{\Delta L}\left[z_{n}(t)-z_{n-1}(t)\right]^{2} .
\end{aligned}
$$

In the above, $-\partial U / \partial z_{n}(t)$ is the force on the nodal point $n$. The results of Sec. II give

$$
\frac{\mathcal{D}}{\Delta L}=\frac{\mathcal{D} N}{L}=D_{\mathrm{COP}} N
$$

and

$$
\frac{\mathcal{C}}{\Delta L}=\frac{\mathcal{C} N}{L}=\frac{k_{b} T N}{12\left\langle|\delta z|^{2}\right\rangle},
$$

which fully defines the Langevin equations of motion to be simulated from the microscopic parameters derived from finite temperature molecular-dynamics simulations performed in the previous section. We note that in the numerical integration of Eq. (44), the chosen time step must be less than 100 fs such 
that the internal dynamics of the loop can be well described. Despite this restriction in the fundamental time scale, due to the several orders of magnitude savings in computational effort (since all atoms of the system are not simulated), simulations of such dislocation loops can be easily performed to the millisecond to seconds time scale thus allowing for direct comparison with experiment.

In performing the above described Langevin dynamics for either the interstitial or vacancy prismatic dislocation loop parameters, measurement of the loop's diffusion constant using the drift-correction method of the Appendix yielded the diffusion coefficients and mean-square fluctuations that were originally put into the model. For the following Langevin simulations, the interstitial data were used. For each temperature a simulation of duration 2 ns was performed using a time step of $10 \mathrm{fs}$. Configuration data were collected every $100 \mathrm{fs}$, as in the case of the molecular-dynamics simulations. The resulting mean-square fluctuations depended linearly on temperature for all temperatures $(100-800 \mathrm{~K})$, falling on a line that intercepted the $T=0 \mathrm{~K}$ origin having a gradient equal to that put into the model [see Figs. 4(b) and 6(b)]. This was performed for three discretizations of the loops using $N=10$, $N=20$, and $N=40$ nodal points. As expected, there is little dependence on the choice of $N$. Figure 8(a) shows the random walks undertaken by the three simulations at $400 \mathrm{~K}$, along with that resulting from the corresponding atomistic simulation. Visually, the structures of all four are indistinguishable. For the case of the Langevin data, the COP diffusion coefficient is independent of the choice of $N$-indeed $N=1$ (a point

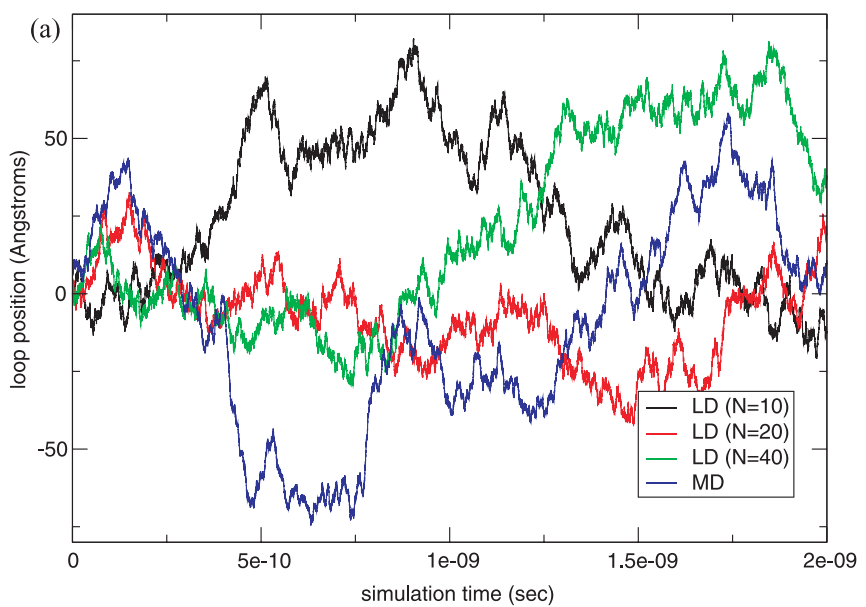

(b)

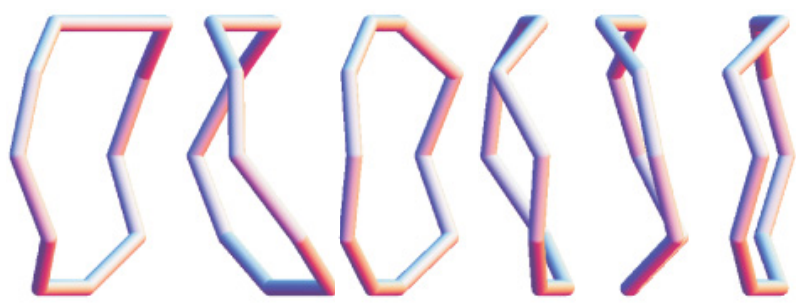

FIG. 8. (Color online) (a) Trajectories of a Langevin dislocation loop using a $N=10,20$, and 40 nodal representation for a given diffusion constant and line tensions derived from molecular-dynamics simulations at $400 \mathrm{~K}$. Also shown is the actual molecular-dynamics trajectory. (b) Sequential snapshots of the $N=10$ Langevin loop. object) would suffice to yield the correct diffusion properties. Figure 8(b) displays a series of snapshots of the $N=10$ dislocation loops, demonstrating the fluctuations occurring during the simulation (six sequential configurations are shown where the time step between each is $100 \mathrm{ps}$ ).

That the above Langevin simulations yielded diffusion coefficients that were precisely those imputed (as a function of temperature, these values are plotted in Figs. 4 and 6 as dotted red lines) demonstrates that the drift-corrected method of measuring diffusion results in extremely wellconverged diffusion coefficients and that the more complex temperature dependence seen in Figs. 4(a) and 6(a) for the molecular-dynamics simulations (when compared to that of literature $^{15-17}$ ) is, in general, not a result of poor trajectory statistics since the Langevin data were purposely chosen to have a sampling equal to that of the molecular-dynamics data. Possible origins of the scatter could be due to a feature of the magnetic potential ${ }^{33}$ used in the simulations or an artifact of extracting the center of position from the high-energy atoms of the molecular-dynamics data.

Finally, it is worth noting that the stochastic differential approach taken here to simulate the elastic string has the great advantage that any temperature-dependent diffusion coefficient model can be used in the Langevin simulationsthus the non-Arrhenius behavior predicted by theory ${ }^{8,9,11}$ and seen in simulation ${ }^{10,11,18}$ can be trivially incorporated into the Langevin dynamics simulations.

\section{DISCUSSION}

In Sec. II, the mean-square displacement of the COP of the elastic loop, Eq. (39), was found to be independent of the elastic stiffness parameter $\mathcal{C}$, whereas the mean-square fluctuation, Eq. (42), depended only on this parameter and not on the diffusion parameter $\mathcal{D}$. This result affords a rather simple picture, which helps to interpret past moleculardynamics simulation work investigating the mobility of SIA clusters, where it was found that the jump rate of SIA clusters had a migration energy barrier that was comparable to an individual SIA and varied only weakly with cluster size. Indeed in the work of Osetsky et al., ${ }^{15}$ SIA cluster movement was described as being facilitated by jumps of individual SIAs. This scenario is precisely the case for the discretized elastic loop considered here, where nodal points (instead of explicit SIAs) have an intrinsic diffusion coefficient (and therefore an intrinsic jump rate) and whose collective diffusion leads directly (and independently of their mutual interaction) to a COP diffusion coefficient that may be identified with that of the cluster's diffusion coefficient. The role of the interaction between such nodal points controls the strength of correlation and therefore the nature of the collective diffusion, but not the actual magnitude of the collective diffusion coefficient, whether it is delocalized (resulting from no interaction) or localized (resulting from interactions) into an object that can be identified as the cluster.

From Eq. (39), $D_{\mathrm{COP}}$ is given by $\mathcal{D} / L \sim \mathcal{D} / \sqrt{A}$, where $A$ is the area of the SIA cluster. Since $A$ is proportional to the number of SIAs within the cluster, $N_{0}$, a natural explanation is given for the observed $N_{0}^{-\alpha}(\alpha \sim 0.5-0.6)$ dependence of 
the prefactor of the jump rate. That the perimeter SIAs are the only active SIAs of the cluster is an essential assumption of the developed model, an assumption that has a strong theoretical grounding in the work of Dudarev ${ }^{24}$ where it was found that platelet SIAs away from the perimeter region become increasingly delocalized to the point that, for large platelet sizes, the instantaneous position of such SIAs becomes ill-defined, effectively decoupling their corresponding degrees of freedom from that of the diffusion dynamics, and relegating them to a strain field that is carried with the SIA cluster.

By performing finite temperature molecular-dynamics simulations, the empirical parameters $\mathcal{D}$ and $\mathcal{C}$ could be determined for both an interstitial and vacancy prismatic loop in bcc Fe. From Figs. 4(a) and 6(a) the values for $\mathcal{D}$ at $300 \mathrm{~K}$ are $\approx L 80 \times 10^{-10} \mathrm{~m}^{2} \mathrm{sec}^{-1}$ for the interstitial loop and $\approx L 18 \times 10^{-10} \mathrm{~m}^{2} \mathrm{sec}^{-1}$ for the vacancy loop, where in both cases the perimeter is $L=57 \AA$. By inspecting the hightemperature gradients of the mean-square internal fluctuations the line tension per unit length for both loops [Figs. 4(b) and 6(b)], $\mathcal{C}$ was determined to be $\approx L 6.0 \times 10^{-4} \mathrm{eV} \AA^{-2}$ for the interstitial loop and $\approx L 6.8 \times 10^{-4} \mathrm{eV} \AA^{-2}$ for the vacancy loop. The uncertainty in both $\mathcal{D}$ and $\mathcal{C}$ is expected to be higher for the case of the vacancy loop due to the difficulty of analyzing its instantaneous position and configuration. With these parameters determined it now becomes possible to model interstitial and vacancy prismatic loops of any size (perimeter) in bcc Fe using the Langevin approach developed in Secs. II and IV, at experimental and human time scales.

The applicable regimes of the developed model are now discussed. There exists a lower loop size in which an elastic continuum description of a prismatic loop is expected to fail. In this regime, $\langle 111\rangle$ crowdions away from the perimeter are not sufficiently delocalized and therefore retain much of the quasiparticle identity of a single $\langle 111\rangle$ crowdion. Such a criterion might actually lead to quite small loops still being described via elasticity theory. Indeed when using the multistring Frenkel-Kontorova model for an edge dislocation via a crowdion representation, the effective spatial extent of each crowdion was found to increase rapidly as a function of distance away from the core of the dislocation structure. ${ }^{24}$ Moreover, in a comparison between atomistic simulation results and continuum elasticity theory, Puigvi et al. ${ }^{25}$ conclude that clusters in bcc metals with diameters above $2 \mathrm{~nm}$ can be described reasonably well as dislocation loops in the elastic continuum approach. The applicability of a continuum elasticity approach is expected to be a minimal requirement of line-tension models such as that developed here.

There also exists an upper loop length scale in which the present elastic string approach is expected to fail. In general, line-tension models have as their central approximation that the local configuration of the dislocation depends on the local properties. Dislocations are, however, topological defects and, as a result, carry a long-range interaction-in principle every point on a dislocation will be influenced by every other point on the same dislocation and those of other dislocations. The underlying approximation of the line-tension picture of the elastic string is that the in-plane kink density (local curvature) is sufficiently high such that the short-range (local) aspect of the kink-kink interaction dominates. With larger loops, the in-plane kink number per unit length decreases and this assumption is expected to break down. Ohsawa and Kuramoto ${ }^{38}$ have calculated the change in energy of a unit fluctuation (defined as a out-of-plane double kink along the loop, with the kink-kink distance being maximal) of a circular loop using a full dislocation-dynamics description for perimeters up to $230 \mathrm{~b}$, and found only a small contribution due to the so-called long-range self-interaction, which increases with loop size. Thus in terms of fluctuations of the loop away from the $0-\mathrm{K}$ perfect circle, the elastic string approximation is expected to be valid. This, however, was done for the highly symmetric circular loop case (as is the case for the present work), and it is expected that such long-range interactions will have an increasing effect in low-symmetry loop configurations, particularly when there exists a population of loops. This is a relevant point, since recent in situ work by Arakawa et al. ${ }^{40}$ has detailed the direct observation of loop coalescence between loops of differing Burgers vector orientation, where the larger loop completely absorbs the smaller loop, therefore changing both its size and shape. It presently remains unclear whether such a complex dislocation geometry and reaction sequence could be described by an extension of the simple line-tension model developed here, or instead by the more contemporary segment-tracking dislocation dynamics simulation method within the framework of the Langevin approach - an avenue of methodology that has already been explored in the study of edge dislocation mobility ${ }^{41}$ and dislocation patterning. ${ }^{42,43}$

In summary, a stochastic differential equation describing a continuum elastic string model has been developed and investigated with the view of developing a simplified description of the collective and internal dynamics of prismatic selfinterstitial and vacancy loops. By exploiting the time-average properties of the stochasticity, relationships between the microscopic parameters of the theory, the friction coefficient, and elastic stiffness per unit length, are given in terms of observables that can be directly obtained from atomistic simulations of prismatic loops. Large scale molecular-dynamics simulations of interstitial and vacancy prismatic loops in bcc Fe have therefore been used to obtain these microscopic empirical parameters resulting in quantitatively analogous Langevin simulations of both the collective and internal dynamics of a prismatic loop at time scales well beyond that achievable with the molecular-dynamics method.

\section{ACKNOWLEDGMENTS}

This work was supported by the EURATOM staff mobility program. Work at CCFE was partly funded by the European Communities under the contract of association between EURATOM and CCFE, and was carried out within the framework of the European Fusion Development Agreement. This work was also partially funded by the RCUK Energy Programme under Grant No. EP/I501045.

\section{APPENDIX: CORRECTED DERIVATION OF DRIFT-CORRECTION METHOD FOR THE MEASUREMENT OF DIFFUSION COEFFICIENTS}

To obtain a diffusion coefficient an ensemble of trajectories is needed from which the mean-squared displacement after a time $t_{0}$ is calculated, $\left\langle z^{2}\left(t_{0}\right)\right\rangle$, directly giving $D$ via the Einstein 
relation $D=\left\langle z^{2}\left(t_{0}\right)\right\rangle / 2 t_{0}$. This relation becomes exact in the limit of large $t_{0}$ since only then is the mean displacement zero. Trajectories obtained from simulation are, however, of finite temporal length. Moreover, a single trajectory is often split into many separate shorter trajectories from which an average of the mean-square displacement is taken under the assumption of statistically independent subtrajectories. ${ }^{44}$ Such short (finite) time trajectories do not have a mean displacement of zero, causing additional statistical fluctuations that make it difficult to obtain a reliable value for the diffusion constant. In the work of Refs. 11 and 34 this fact was noticed and corrected for by subtracting off the actual displacement of each subtrajectory before taking the ensemble average, resulting in a better estimate of the corresponding diffusion coefficient.

The more reliable diffusion coefficient was obtained via

$$
D_{\text {measured }}=\frac{1}{2 t_{0}}\left\langle\left(z\left(t_{0}\right)-\frac{2}{t_{0}} \int_{0}^{t_{0}} d \tau z(\tau)\right)^{2}\right\rangle,
$$

where the second term within the square is the so-called drift correction. Expanding the square within the average gives

$$
\begin{aligned}
D_{\text {measured }}= & \frac{1}{2 t_{0}}\left(\left\langle z^{2}\left(t_{0}\right)\right\rangle-\frac{4}{t_{0}} \int_{0}^{t_{0}} d \tau\left\langle z\left(t_{0}\right) z(\tau)\right\rangle\right. \\
& \left.+\frac{4}{t_{0}^{2}} \int_{0}^{t_{0}} d \tau \int_{0}^{t_{0}} d \tau^{\prime}\left\langle z(\tau) z\left(\tau^{\prime}\right)\right\rangle\right),
\end{aligned}
$$

resulting in the diffusion coefficient being a function of the mean-square displacement and also the position-position correlation function-when finite time trajectories are employed. For sufficiently large $t_{0}$ it was assumed in Refs. 11 and 34 that these correlation functions would vanish and the drift corrected $D_{\text {measured }}$ would be formally identical to the actual diffusion coefficient. However, the present simulations (both molecular dynamics and Langevin dynamics) have shown that this is not the case. Indeed, when applying the drift correction method, diffusion coefficients were obtained that were a factor of 3 larger than that obtained when using a non-drift-correction method.

This result can be understood by explicitly calculating the above correlation functions within the framework of a one- dimensional Langevin equation. The overdamped Langevin equation in one dimension can be written as

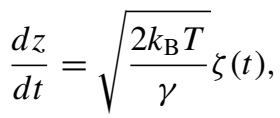

where $D=k_{\mathrm{B}} T / \gamma$ and

$$
\left\langle\zeta(t) \zeta\left(t^{\prime}\right)\right\rangle=\delta\left(t-t^{\prime}\right)
$$

Equation (A3) has the solution

$$
z(t)=\sqrt{\frac{2 k_{\mathrm{B}} T}{\gamma}} \int_{0}^{t} d \tau \zeta(\tau)
$$

giving the position-position correlation function as

$$
\begin{aligned}
\left\langle z(t) z\left(t^{\prime}\right)\right\rangle & =\frac{2 k_{\mathrm{B}} T}{\gamma} \int_{0}^{t} d \tau \int_{0}^{t^{\prime}} d \tau^{\prime}\left\langle\zeta(\tau) \zeta\left(\tau^{\prime}\right)\right\rangle \\
& =\frac{2 k_{\mathrm{B}} T}{\gamma} \int_{0}^{t} d \tau \int_{0}^{t^{\prime}} d \tau^{\prime} \delta\left(\tau-\tau^{\prime}\right) .
\end{aligned}
$$

By using the fact that if $t>t^{\prime}$, the integral

$$
\int_{0}^{t^{\prime}} d \tau^{\prime} \delta\left(\tau-\tau^{\prime}\right)
$$

equals zero if $\tau>t^{\prime}$ and unity if $\tau<t^{\prime}$, and always unity if $t<t^{\prime}$, the position-position correlation function can be shown to reduce to

$$
\left\langle z(t) z\left(t^{\prime}\right)\right\rangle=\frac{2 k_{\mathrm{B}} T}{\gamma} \min \left\{t, t^{\prime}\right\} .
$$

Thus the position-position correlation function does not vanish in the $\left|t-t^{\prime}\right| \rightarrow \infty$ limit. This was confirmed via simulation. Figure 9 displays the correlation function, $\left\langle z\left(t_{0}\right) z(\tau)\right\rangle$, derived from atomistic and Langevin simulations of a interstitial cluster at $400 \mathrm{~K}$, and it is seen that for large $t$ and $t^{\prime}$ it grows linearly. After some integration and algebra, Eq. (A9) results in the measured diffusion constant [Eq. (A2)] equaling

$$
D_{\text {measured }}=\frac{k_{\mathrm{B}} T}{\gamma}-\frac{2 k_{\mathrm{B}} T}{\gamma}+\frac{4 k_{\mathrm{B}} T}{3 \gamma}=\frac{1}{3} \frac{k_{\mathrm{B}} T}{\gamma}=\frac{D}{3},
$$
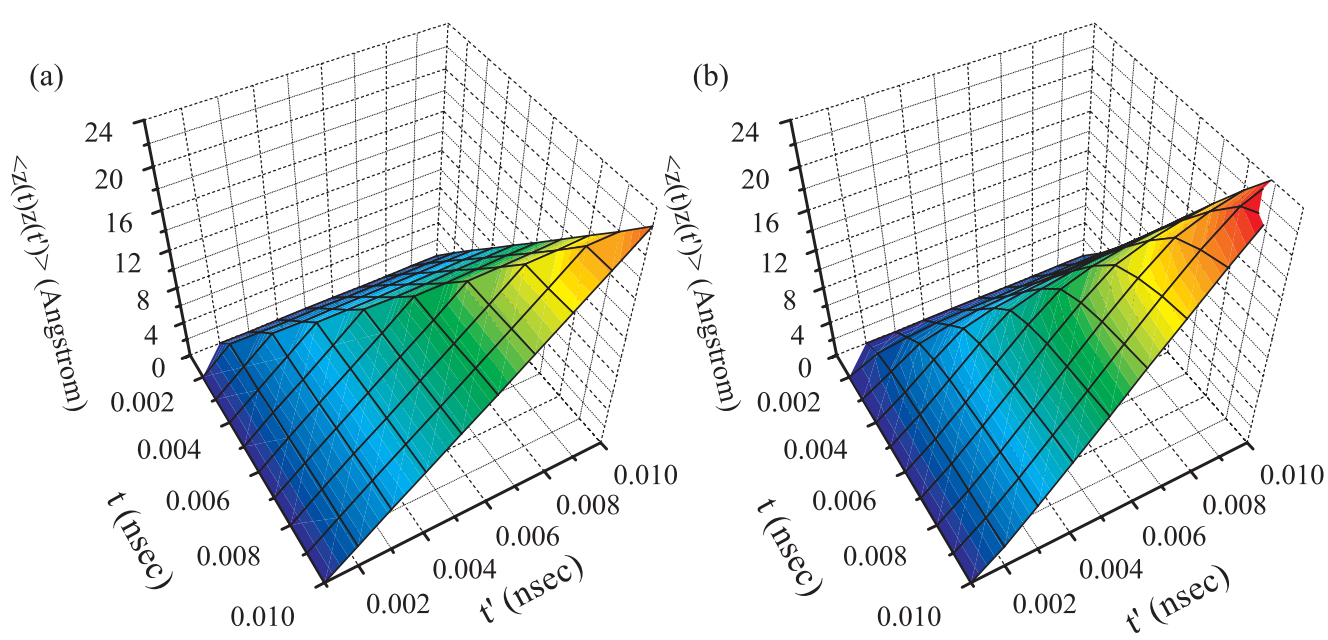

FIG. 9. (Color online) The measured correlation function $\left\langle z(t) z\left(t^{\prime}\right)\right\rangle$ obtained via (a) molecular and (b) Langevin dynamics. 
which explains the origin of the discrepancy in the obtained diffusion coefficient. Thus although the drift-correction method achieves more reliable values of the diffusion constant, the physical diffusion coefficient is one-third of the obtained value and therefore the correcting factor of 3 needs to be introduced in the drift-correcting equations given in Refs. 11 and 34. *peter.derlet@psi.ch

${ }^{1}$ S. L. Dudarev, J.-L. Boutard, R. Lässer, M. J. Caturla, P. M. Derlet, M. Fivel, C.-C. Fu, M. Y. Lavrentiev, L. Malerba, M. Mrovec, D. Nguyen-Manh, K. Nordlund, M. Perlado, R. Schäublin, H. Van Swygenhoven, D. Terentyev, J. Wallenius, D. Weygand, and F. Willaime, J. Nucl. Mater. 386-388, 1 (2009).

${ }^{2}$ C. Domain and C. S. Becquart, Phys. Rev. B 65, 024103 (2001).

${ }^{3}$ C. C. Fu, F. Willaime, and P. Ordejón, Phys. Rev. Lett. 92, 175503 (2004).

${ }^{4}$ C. C. Fu, J. Dalla Torre, F. Willaime, J. L. Bocquet, and A. Barbu, Nat. Mater. 4, 68 (2005).

${ }^{5}$ D. Nguyen-Manh, A. P. Horsfield, and S. L. Dudarev, Phys. Rev. B 73, 020101 (2006).

${ }^{6}$ B. D. Wirth, G. R. Odette, D. Maroudas, and G. E. Lucas, J. Nucl. Mater. 276, 33 (2000).

${ }^{7}$ Y. N. Osetsky, D. J. Bacon, A. Serra, B. N. Singh, and S. I. Golubov, J. Nucl. Mater. 276, 65 (2000).

${ }^{8}$ S. L. Dudarev, J. Nucl. Mater. 307-311, 881 (2002).

${ }^{9}$ S. L. Dudarev, Phys. Rev. B 65, 224105 (2002).

${ }^{10}$ L. A. Zepeda-Ruiz, J. Rottler, S. Han, G. J. Ackland, R. Car, and D. J. Srolovitz, Phys. Rev. B 70, 060102(R) (2004).

${ }^{11}$ P. M. Derlet, D. Nguyen-Manh, and S. L. Dudarev, Phys. Rev. B 76, 054107 (2007).

${ }^{12}$ R. A. Johnson, Phys. Rev. 134, A1329 (1964).

${ }^{13}$ B. D. Wirth, G. R. Odette, D. Maroudas, and G. E. Lucas, J. Nucl. Mater. 244, 185 (1997).

${ }^{14}$ S. Chiesa, P. M. Derlet, S. L. Dudarev, and H. Van Swygenhoven, J. Phys.: Condens. Matter 23, 206001 (2011).

${ }^{15}$ Y. N. Osetsky, D. J. Bacon, A. Serra, B. N. Singh, and S. I. Golubov, Philos. Mag. 83, 61 (2003).

${ }^{16}$ D. A. Terentyev, L. Malerba, and M. Hou, Phys. Rev. B 75, 104108 (2007).

${ }^{17}$ N. Anento, A. Serra, and Y. N. Osetsky, Modell. Simul. Mater. Sci. Eng. 18, 025008 (2010).

${ }^{18}$ L. A. Zepeda-Ruiz, J. Rottler, B. D. Wirth, R. Car, and D. J. Srolovitz, Acta Mater. 53, 1985 (2005).

${ }^{19}$ F. Willaime, C. C. Fu, M. C. Marinca, and J. Dalla Torre, Nucl. Instrum. Methods Phys. Res. B 228, 92 (2005).

${ }^{20}$ P. M. Derlet and S. L. Dudarev, Prog. Mater. Sci. 52, 299 (2007).

${ }^{21}$ M. R. Gilbert, S. L. Dudarev, P. M. Derlet, and D. G. Pettifor, J. Phys.: Condens. Matter 20, 345214 (2008).
${ }^{22}$ S. L. Dudarev, R. Bullough, and P. M. Derlet, Phys. Rev. Lett. 100, 135503 (2008).

${ }^{23}$ The jump frequency is in general a more easily determined quantity than the diffusion coefficient since it requires only the sampling of the time between migration events of the diffusing defect. An Arrhenius behavior of this quantity does not at all imply a similar behavior for the diffusion coefficient and it should be noted that in the case of fast interstitial migration where the obtained migration barriers are comparable to the thermal energy scale, the assumption of a "rare event" phenomenon (central to the thermal activation hypothesis underlying the Arrhenius form) is not satisfied.

${ }^{24}$ S. L. Dudarev, Philos. Mag. 83, 3577 (2003).

${ }^{25}$ M. A. Puigvi, Y. N. Osetsky, and A. Serra, Philos. Mag. 83, 857 (2003).

${ }^{26} \mathrm{~K}$. Arakawa, K. Ono, M. Isshiki, K. Mimura, M. Uchikoshi, and H. Mori, Science 318, 956 (2007).

${ }^{27}$ M. L. Jenkins, Z. Yao, M. Hernandez-Mayoral, and M. A. Kirk, J. Nucl. Mater. 389, 197 (2009).

${ }^{28}$ Z. Yao, M. Hernandez-Mayoral, M. L. Jenkins, and M. A. Kirk, Philos. Mag. 88, 2851 (2008).

${ }^{29}$ M. Hernandez-Mayoral, Z. Yao, M. L. Jenkins, and M. A. Kirk, Philos. Mag. 88, 2881 (2008).

${ }^{30}$ Y. Matsukawa and S. J. Zinkle, Science 318, 959 (2007).

${ }^{31}$ M. J. Caturla, N. Soneda, E. Alonso, B. D. Wirth, T. D. de la Rubia, and J. M. Perlado, J. Nucl. Mater. 276, 13 (2000).

${ }^{32}$ S. L. Dudarev, M. R. Gilbert, K. Arakawa, H. Mori, Z. Yao, M. L. Jenkins, and P. M. Derlet, Phys. Rev. B 81, 224107 (2010).

${ }^{33}$ S. L. Dudarev and P. M. Derlet, J. Phys.: Condens. Matter 17, 7097 (2005).

${ }^{34}$ S. L. Dudarev, C. R. Phys. 9, 409 (2009).

${ }^{35}$ A. V. Granato and K. Lücke, J. Appl. Phys. 27, 583 (1956).

${ }^{36}$ A. D. Brailsford, Phys. Rev. 128, 1033 (1962).

${ }^{37}$ A. D. Brailsford, J. Appl. Phys. 36, 3941 (1965).

${ }^{38}$ K. Ohsawa and E. Kuramoto, Phys. Rev. B 72, 054105 (2005).

${ }^{39}$ K. Ohsawa and E. Kuramoto, J. Nucl. Mater. 367-370, 327 (2007).

${ }^{40}$ K. Arakawa, T. Amino, and H. Mori, Acta Mater. 59, 141 (2011).

${ }^{41}$ M. Hiratani and H. M. Zbib, J. Eng. Mater. Tech.-Trans. ASME 124, 335 (2002).

${ }^{42}$ B. Bako and I. Groma, Phys. Rev. B 60, 122 (1999).

${ }^{43}$ M. Hiratani and H. M. Zbib, J. Nucl. Mater. 323, 290 (2003).

${ }^{44}$ M. W. Guinan, R. N. Stuart, and R. J. Borge, Phys. Rev. B 15, 699 (1977). 